\title{
Patterns of Use and Correlates of Patient Satisfaction with a Large Nationwide Direct to Consumer Telemedicine Service
}

\author{
Kathryn A. Martinez, PhD, MPH' , Mark Rood, MD², Nikhyl Jhangiani, MBA, MPH ${ }^{3}$, Lei Kou, M.A. ${ }^{4}$, \\ Susannah Rose, PhD, MSSW' ${ }^{5}$, Adrienne Boissy, $\mathrm{MD}^{5}$, and Michael B. Rothberg, MD, MPH
}

${ }^{1}$ Center for Value-Based Care Research, Cleveland Clinic, Cleveland, OH, USA; ${ }^{2}$ Department of Family Medicine, Cleveland Clinic, Cleveland, $\mathrm{OH}$, USA; ${ }^{3}$ Distance Health, Cleveland Clinic, Cleveland, OH, USA; ${ }^{4}$ Department of Quantitative Health Sciences, Cleveland Clinic, Cleveland, OH, USA; ${ }^{5}$ Office of Patient Experience, Cleveland Clinic, Cleveland, OH, USA.

BACKGROUND: Despite its rapid expansion, little is known about use of direct to consumer (DTC) telemedicine. OBJECTIVE: To characterize telemedicine patients and physicians and correlates of patient satisfaction

DESIGN: Cross-sectional study

PARTICIPANTS: Patients and physicians of a large nationwide DTC telemedicine service

MAIN MEASURES: Patient characteristics included demographics and whether or not they reported insurance information. Physician characteristics included specialty, board certification, and domestic versus international medical training. Encounter characteristics included time of day, wait time, length, coupon use for free or reducedcost care, diagnostic outcome, prescription receipt, and patient/physician geographic concordance. Patients rated satisfaction with physicians on scales of 0 to 5 stars and reported where they would have sought care had they not used telemedicine. Logistic regression was used to assess factors associated with 5-star physician ratings.

KEY RESULTS: The analysis included 28,222 encounters between 24,040 patients and 277 physicians completed between January 2013 and August 2016. Sixty-five percent of patients were under 40 years and 32\% did not report insurance information. Family medicine was the most common physician specialty (47\%) and $16 \%$ trained at a non-US medical school. Coupons were used in $24 \%$ of encounters. Respiratory infections were diagnosed in $35 \%$ of encounters and $69 \%$ resulted in a prescription. Had they not used telemedicine, $43 \%$ of patients reported they would have used urgent care/retail clinic, 29\% would have gone to the doctor's office, $15 \%$ would have done nothing, and 6\% would have gone to the emergency department. Eighty-five percent of patients rated their physician 5 stars. High satisfaction was positively correlated with prescription receipt (OR 2.98; 95\%CI 2.743.23) and coupon use (OR 1.47; 95\%CI 1.33-1.62).

CONCLUSIONS: Patients were largely satisfied with DTC telemedicine, yet satisfaction varied by coupon use and prescription receipt. The impact of telemedicine on primary care and emergency department use is likely to be small under present usage patterns.

KEY WORDS: telemedicine; consumer health; patient satisfaction.

Received February 26, 2018

Revised July 10, 2018

Accepted July 31, 2018

Published online August 15, 2018
J Gen Intern Med 33(10):1768-73

DOI: $10.1007 / \mathrm{s} 11606-018-4621-5$

(C) Society of General Internal Medicine 2018

\section{INTRODUCTION}

Direct to consumer (DTC) telemedicine has been touted as an opportunity to expand access while reducing costs, ${ }^{1}$ by allowing patients access to medical care $24 \mathrm{~h}$ a day, via web and phone-enabled virtual visits. Use of DTC telemedicine is expanding rapidly, ${ }^{2}$ growing from approximately 2 million primary care video consultations in 2015 to more than 5 million projected by $2020 .^{3}$

Given current demands on the healthcare system, the rapid expansion of DTC telemedicine is unsurprising. It takes an average of 20 days to secure a new appointment with a physician, ${ }^{4}$ and more than half US adults report being unable to get a same or next day appointment with their doctor. ${ }^{5}$ Opportunity costs associated with seeking care in the USA are substantial and often exceed out of pocket costs. ${ }^{6}$ The average outpatient visit requires $121 \mathrm{~min}$, including $37 \mathrm{~min}$ of travel time and $84 \mathrm{~min}$ of in-clinic time, ${ }^{6}$ with only $15 \mathrm{~min}$ spent with the physician. ${ }^{7}$ The growing use of retail clinics in recent years ${ }^{8}$ reflects consumer demand for more convenient ways to access care. ${ }^{9}$ Yet telemedicine has a critical advantage over retail clinics: the ability of patients to access medical care without leaving home or work.

Another advantage of DTC telemedicine is cost. Without physical overhead, medical assistants, and billing personnel, telemedicine has a per-episode cost of care below that provided in brick and mortar settings. ${ }^{10}$ While insurance coverage for telemedicine has not kept pace with its expansion, 32 states currently have telehealth parity laws requiring private insurers to cover telemedicine services, ${ }^{11}$ and this is projected to grow. In the meantime, the low out of pocket cost of a DTC telemedicine visit makes it an attractive alternative for those without insurance. Indeed, many DTC telemedicine patients have little prior experience with the healthcare system. ${ }^{12}$

Despite its rapid expansion, much is still unknown about DTC telemedicine, including who is using it and for what purpose. Information on telemedicine physicians is similarly scant. While some studies have found that most patients are 
satisfied with telemedicine, these studies were conducted in limited populations, restricted to insured individuals from specific health plans or medical practices. ${ }^{13,14}$ Consequently, patient satisfaction with DTC telemedicine and its correlates are unknown. ${ }^{15}$

The objectives of this study were to characterize patients, physicians, and use of a large nationwide DTC telemedicine service and to explore correlates of patient satisfaction with their telemedicine physicians.

\section{METHODS}

This study uses data from the Online Care Group (OCG), a large nationwide DTC telemedicine provider that operates on American Well's software, a HIPAA-compliant, encrypted platform. The OCG connects patients with physicians $24 \mathrm{~h}$ a day to address minor, urgent medical problems through synchronous video visits. The OCG is one of numerous DTC telemedicine platforms; similar platforms include MD Live or Doctor on Demand. The OCG works with payers, health systems, and employer groups to deliver and market services.

When patients access the OCG system, the interface first collects patient information, followed by insurance information. For those who do not have insurance or do not wish to report insurance information, patients can pay out of pocket. During the study period, a typical visit cost $\$ 49$. The system then prompts the patient to describe their reason for seeking care via a free-text field before being placed in a queue for the next available provider.

OCG physicians are recruited directly using traditional job sites and trade publications and are not associated with any specific health or hospital system. Some OCG physicians are salaried and others are paid by encounter or by the hour. Physicians are a mix of full-time, part-time, contract, and members of other medical groups contracted to work with OCG. The average OCG physician works about $10 \mathrm{~h}$ a week on the platform. However, some physicians work as much as $80 \mathrm{~h}$ a week for the OCG.

In June 2015, Cleveland Clinic entered an agreement to use the American Well platform for its online healthcare needs. Consistent with its academic research focus, Cleveland Clinic then negotiated a data use agreement to allow a descriptive analysis of the OCG raw data. These data are platform-wide and not specific to Cleveland Clinic. This study describes OCG encounters completed with physicians between January 1, 2013 and August 31, 2016.

This study was approved by the Institutional Review Board of the Cleveland Clinic.

\section{Measures}

Patient Characteristics. Telemedicine patients provided age, sex, and insurance information for billing purposes. We dichotomized insurance information as "provided insurance information" versus not. Geographic region was determined by patient-reported US state, which we grouped into Censusdefined regions. Some patients accessed the telemedicine system multiple times during the study period. We dichotomized usage as once versus two or more times.

Coupon Use. To increase uptake, some employers and health plans offer coupons for free or reduced-cost telemedicine encounters. Whether or not the patient provided a coupon code was recorded by the system.

Encounter Characteristics. Encounter characteristics were recorded by the system. Call time of day was categorized as 12 am to 7 am, 7 am to $5 \mathrm{pm}$, and $5 \mathrm{pm}$ to $12 \mathrm{am}$. Wait time was defined as the time between the patient being placed in the queue and when they were connected with a physician. Encounter length was defined as the time between the start and completion of the encounter with the physician. Wait time and encounter length were recorded in minutes.

Diagnostic Outcome. Telemedicine physicians recorded diagnoses (up to six) using ICD-9 and ICD-10 codes. We assessed the 220 most common codes. Two primary care physicians grouped the codes into the following clinical categories: respiratory, chronic disease, urinary, dermatological, mental health, gastrointestinal, musculoskeletal/rheumatologic, genital, eye and ear, neurological, asthma, or other. Diagnostic categories were analyzed as independent dichotomous variables (e.g., respiratory diagnosis versus not). As some encounters did not have any associated ICD codes, we also assessed whether encounters resulted in any diagnosis versus not as a separate variable.

Medication Prescriptions. Whether or not the patient received a prescription was determined by whether the physician recorded a National Drug Code (NDC) for the encounter.

Patient Satisfaction. At encounter conclusion, patients were prompted to rate their satisfaction with their telemedicine physician on scales of 0 to 5 stars, with 5 stars being most satisfied. We dichotomized responses as 5 stars versus less than 5 stars, consistent with analysis of other patient satisfaction measures. ${ }^{16}$

Where Patients Would Have Sought Care Otherwise. A major question related to telemedicine is whether it is a complement or a substitute of in-person care. At encounter conclusion, patients were prompted with the question "If you had not used online care today, where would you have gone instead?" Response categories included urgent care/retail clinic, emergency department, doctor's office, or done nothing. 
Physician Characteristics. For each encounter, the OCG provided first and last names of physicians. Through publicly available sources, we determined physician specialty and whether they were board certified. Physician state of residence was reported by the OCG, which we categorized into Census-defined regions. Additionally, to account for possible non-native English speaking or cultural factors which might influence patient satisfaction, we determined whether physicians trained at a US or non-US medical school.

\section{Regional Concordance Between Patient and Physician.}

Telemedicine is unique in that it pairs physicians and patients from different parts of the country. Regional variation in medical care is well-established ${ }^{17}$ and may influence patient expectations. Thus, we assessed whether regional concordance between patients and physicians was associated with satisfaction with care.

\section{Analysis}

We characterized telemedicine patients, physicians, and encounters by the characteristics described above. We described patient satisfaction with telemedicine physicians, as well as what patients said they would have done had they not accessed the telemedicine system. Using mixed effects logistic regression, we assessed differences in patient satisfaction with telemedicine physicians by the factors described above, accounting for clustering by physician. Analyses were conducted in Stata 14.

\section{RESULTS}

Patient and physician characteristics are presented in Table 1. There were 28,222 completed encounters between 24,040 patients and 277 physicians during the study period. Fiftynine percent of patients were female and $55 \%$ were between the ages of 18 and 40 years. A third of patients (34\%) were from the South, followed by Midwest (26\%), West (25\%), and Northeast (15\%). Eighty-seven percent of patients accessed the system once and $13 \%$ accessed it two or more times. Most physicians (84\%) trained at a US medical school and $47 \%$ specialized in family medicine, followed by internal medicine (20\%). Almost all physicians (91\%) were board certified. Physicians were geographically concentrated in the South $(30 \%)$ and West $(30 \%)$. Over the study period, the mean number of encounters per physician was 101 , the median was 11 , and the range was 1 to 1774 . Fifty percent of physicians completed 11 or fewer encounters during the study period and only 21 physicians completed 500 or more.

Encounter characteristics and patient satisfaction responses are presented in Table 2. Utilization increased over the study period, with 157 encounters in 2013, 828 in 2015, 6545 in 2015, and 20,692 in 2016. Most (60\%) encounters were
Table 1 Patient and Physician Characteristics

\begin{tabular}{ll}
\hline \hline & $N(\%)$ \\
\hline Patient characteristics $(N=24,040)$ & \\
Female & $14,178(59)$ \\
Age (years) & \\
$<18$ & $2474(10)$ \\
$18-29$ & $5734(24)$ \\
$30-39$ & $7535(31)$ \\
$40-49$ & $4790(20)$ \\
50 and over & $3507(15)$ \\
Provided insurance information & $16,324(68)$ \\
Geographic region & \\
Northeast & $3608(15)$ \\
Midwest & $6148(26)$ \\
South & $8183(34)$ \\
West & $6092(25)$ \\
Number of telemedicine visits during study period & \\
One & $20,877(87)$ \\
Two or more & $3163(13)$ \\
Physician characteristics $(N=277)$ & \\
Specialty & \\
Family medicine & $129(47)$ \\
Internal medicine & $55(20)$ \\
Emergency medicine & $47(17)$ \\
Pediatrics & $23(8)$ \\
Other & $23(8)$ \\
Board certified & $252(91)$ \\
Trained at a non-US medical school & $43(16)$ \\
Geographic region & $66(24)$ \\
Northeast & $44(16)$ \\
Midwest & $83(30)$ \\
South & $84(30)$ \\
West & $101(1-1774)$ \\
Number of encounters per physician, mean(range) & \\
\hline & \\
\hline & \\
\hline
\end{tabular}

Table 2 Encounter Characteristics and Patient-Reported Measures

\begin{tabular}{ll}
\hline \hline Encounter characteristics $(N=28,222)$ & \\
Number of encounters, by year, $N(\%)$ & $157(1)$ \\
2013 & $828(3)$ \\
2014 & $6545(23)$ \\
2015 & $20,692(73)$ \\
2016 & \\
Encounter time of day, $N(\%)$ & $1753(6)$ \\
12 am to 7 am & $17,032(60)$ \\
7 am to 5 pm & $9437(33)$ \\
5 pm to 12 am & $10,809(38)$ \\
Patient and physician geographic concordance, $N(\%)$ & $5.3(1.2-6.4)$ \\
Wait time (min), mean (IQR) & $6.6(3.7-8.6)$ \\
Encounter length (min), mean (IQR) & $6767(24)$ \\
Used coupon, $N(\%)$ & $19,495(69)$ \\
Received prescription, $N(\%)$ & \\
Encounter diagnosis, $N(\%)$ & $9839(35)$ \\
Respiratory & $2523(9)$ \\
Urinary tract & $1878(7)$ \\
Other & $2037(7)$ \\
Eye and ear & $1455(5)$ \\
Dermatological & $679(2)$ \\
Mental health & $539(2)$ \\
Gastrointestinal & $551(2)$ \\
Genital & $457(2)$ \\
Chronic & $251(1)$ \\
Musculoskeletal or rheumatologic & $285(1)$ \\
Asthma & $156(0.5)$ \\
Neurological & $1607(6)$ \\
Did not receive diagnosis, $N(\%)$ & \\
Patient-reported measures & \\
Satisfaction with provider, $N(\%)$ & $23,974(85)$ \\
Five stars & $1566(6)$ \\
Where patients would have sought care otherwise, $N(\%)$ & $8224(29)$ \\
Emergency department & $13,662(43)$ \\
Doctor's office & $4810(15)$ \\
Urgent care/retail clinic & \\
Done nothing & \\
\hline & \\
&
\end{tabular}


during daytime working hours (7 am to $5 \mathrm{pm}$ ). The average wait time was $5.3 \mathrm{~min}$ (interquartile range (IQR) 1.2-6.4) and the average encounter length was $6.6 \mathrm{~min}$ (IQR 3.7-8.6). Patients provided coupon codes for a free or reduced-cost visit in $24 \%$ of encounters. Among those patients who did not report insurance information, $44 \%$ used a coupon, compared to $14 \%$ of patients who reported insurance information. The most common diagnoses were respiratory issues (35\%), urinary issues $(9 \%)$, eye and ear problems (7\%), and dermatological conditions (5\%). Six percent of encounters did not result in any diagnosis and 69\% ended with a prescription, $67 \%$ of which were for an antibiotic. Overall, $85 \%$ of patients rated their satisfaction with their telemedicine physician five stars. Had they not used the telemedicine system that day, $43 \%$ of patients said they would have gone to urgent care or a retail clinic, $29 \%$ said they would have gone to the doctor's office, $15 \%$ would have done nothing, and $6 \%$ would have gone to the ED.

In the adjusted logistic model of patient satisfaction with their telemedicine physician (Table 3), prescription receipt (OR 2.98; 95\%CI 2.74-3.23) and coupon use (OR 1.47; 95\%CI 1.33-1.62) were associated with higher odds of patients rating their physician five stars. Relative to 18-29 year olds, 30-39 year olds rated their physician more highly (OR $1.21 ; 95 \%$ CI $1.10-1.33$ ), as did users calling on behalf of pediatric patients $(<18$ years) (OR: 1.25 ; 95\%CI $1.09-1.43$ ). Patients who used the telemedicine system two or more times during the study period rated their physicians more highly versus those who used it only once (OR 1.13; 95\%CI 1.041.23). Compared to encounters between 12 am and $7 \mathrm{am}$, those between 7 am and 5 pm (OR 1.23; 95\%CI 1.06-1.43) and $5 \mathrm{pm}$ to 12 am (OR 1.20; 95\%CI 1.03-1.41) had higher ratings. Encounters with non-US trained physicians were less likely to result in five-star ratings (OR 0.71 ; 95\% CI 0.53 0.94). Longer wait time was associated with lower satisfaction (OR per minute 0.99 ; 95\% CI 0.99-0.99), as was not receiving any diagnosis (OR 0.52 ; 95\% CI $0.45-0.60)$. Encounters that resulted in a diagnosis for a urinary issue (OR 1.36 ; $95 \% \mathrm{CI}$ 1.14-1.62), asthma (OR 1.82; 95\%CI 1.07-3.11), or chronic condition (OR 1.58; 95\%CI 1.08-2.30) had higher physician ratings, while those resulting in dermatological (OR 0.75; 95\%CI 0.63-0.89), musculoskeletal/rheumatologic (OR 0.70 ; 95\%CI 0.49-0.98), or "other" diagnoses (OR 0.51; $95 \% \mathrm{CI} 0.44-0.58)$ were less likely to result in a five-star rating Also of note were factors that not associated with patient satisfaction, including physician region, specialty or board certification, patient sex, reporting insurance information or region, encounter length, or geographical concordance between patient and physician.

\section{DISCUSSION}

In our study of a large nationwide DTC telemedicine service, use increased rapidly over the study period. Patients were
Table 3 Mixed Effects Logistic Regression, Odds of Rating Physician Five Stars

\begin{tabular}{|c|c|c|}
\hline & OR & $95 \% \mathrm{CI}$ \\
\hline \multicolumn{3}{|l|}{ Patient sex } \\
\hline Male (versus female) & 1.03 & $0.96-1.11$ \\
\hline \multicolumn{3}{|l|}{ Patient age (years) } \\
\hline $18-29$ & 1.00 & \\
\hline $30-39$ & 1.21 & $1.10-1.33$ \\
\hline $40-49$ & 0.98 & $0.89-1.09$ \\
\hline 50 and over & 1.03 & $0.89-1.13$ \\
\hline$<18$ (pediatric) & 1.25 & $1.09-1.43$ \\
\hline \multicolumn{3}{|l|}{ Patient region } \\
\hline Northeast & 1.00 & \\
\hline Midwest & 0.86 & $0.77-0.97$ \\
\hline South & 1.03 & $0.92-1.16$ \\
\hline West & 1.05 & $0.93-1.20$ \\
\hline Provided insurance information (versus not) & 0.98 & $0.90-1.06$ \\
\hline \multicolumn{3}{|l|}{ Number of visits } \\
\hline One & 1.00 & \\
\hline Two or more & 1.13 & $1.04-1.23$ \\
\hline \multicolumn{3}{|l|}{ Call time of day } \\
\hline 12 am to 7 am & 1.00 & \\
\hline $7 \mathrm{am}$ to $5 \mathrm{pm}$ & 1.23 & $1.06-1.43$ \\
\hline $5 \mathrm{pm}$ to $12 \mathrm{am}$ & 1.20 & $1.03-1.41$ \\
\hline Used coupon (versus not) & 1.47 & $1.33-1.62$ \\
\hline Received prescription (versus not) & 2.98 & $2.74-3.23$ \\
\hline $\begin{array}{l}\text { Did not receive any diagnosis } \\
\text { (versus diagnosis receipt) }\end{array}$ & 0.52 & $0.45-0.60$ \\
\hline \multicolumn{3}{|l|}{ Diagnoses (versus not) } \\
\hline Respiratory & 0.85 & $0.77-0.94$ \\
\hline Urinary tract & 1.36 & $1.14-1.62$ \\
\hline Eye and ear & 0.87 & $0.74-1.03$ \\
\hline Dermatological & 0.75 & $0.63-0.89$ \\
\hline Mental health & 0.99 & $0.76-1.28$ \\
\hline Gastrointestinal & 0.90 & $0.70-1.15$ \\
\hline Chronic & 1.58 & $1.08-2.30$ \\
\hline Genital & 0.98 & $0.73-1.31$ \\
\hline Musculoskeletal or rheumatologic & 0.70 & $0.49-0.98$ \\
\hline Asthma & 1.82 & $1.07-3.11$ \\
\hline Neurological & 0.73 & $0.47-1.13$ \\
\hline Other & 0.51 & $0.44-0.58$ \\
\hline Wait time, per minute & 0.99 & $0.99-0.99$ \\
\hline Encounter length, per minute & 1.00 & $0.99-1.01$ \\
\hline \multicolumn{3}{|l|}{ Physician specialty } \\
\hline Internal medicine & 1.00 & \\
\hline Family medicine & 0.99 & $0.75-1.32$ \\
\hline Emergency medicine & 1.09 & $0.74-1.60$ \\
\hline Pediatrics & 0.79 & $0.49-1.29$ \\
\hline Other & 1.49 & $0.89-2.50$ \\
\hline Not board certified (versus board certified) & 0.84 & $0.60-1.17$ \\
\hline Non-US trained (versus US trained) & 0.71 & $0.53-0.94$ \\
\hline \multicolumn{3}{|l|}{ Physician region } \\
\hline Northeast & 1.00 & \\
\hline Midwest & 0.87 & $0.68-1.13$ \\
\hline South & 0.98 & $0.76-1.25$ \\
\hline West & 1.09 & $0.85-1.41$ \\
\hline $\begin{array}{l}\text { Patient/physician regional concordance } \\
\text { (versus discordance) }\end{array}$ & 1.01 & $0.94-1.10$ \\
\hline
\end{tabular}

young and the majority was female. Family medicine was the most common specialty among telemedicine physicians and nearly all were US-trained. respiratory diagnoses were most common, followed by urinary issues, eye and ear problems, and dermatological conditions. Satisfaction with our sample of telemedicine physicians was high. Patients who received a prescription and those who used a coupon were more satisfied, while patients did not receive a diagnosis were less satisfied. According to patient self-report, use of the OCG telemedicine service appears to serve primarily as a substitute for urgent care/retail clinics and, to a lesser extent, physician visits. 
Prior telemedicine studies have largely been isolated to a particular medical practice ${ }^{18}$ or insurance plan. ${ }^{10,12}$ To our knowledge, ours is the first to characterize DTC telemedicine patients and use in a large geographically diverse population, irrespective of insurance status or employment. To date, the largest prior study of DTC telemedicine patients and use was an analysis of 4657 visits to the Teladoc system by individuals insured through the California Public Employees Retirement System. ${ }^{12}$ The Teladoc system differs from the OCG in that patients first request an appointment, at which point a physician reviews the patient's medical history before getting in touch with the patient about $20 \mathrm{~min}$ later via telephone. Similar to this Teladoc study, we found the largest proportion of patients were diagnosed with acute respiratory conditions. However, the authors of the Teladoc study noted that there was a wide variety of conditions for which patients sought care. This was also true in our study population. In fact, we found over 3000 unique diagnostic codes. Retail clinics, in comparison, address a much narrower array of healthcare needs. ${ }^{19}$ The convenience of being able to address a health concern in a very short amount of time without needing to leave home or work likely lowers the threshold for care seeking, increasing the diversity of diagnoses. Perhaps the semi-anonymous nature of DTC telemedicine also motivates patients to seek care for issues they would not have otherwise.

The potential beneficial impact of DTC telemedicine on healthcare spending has garnered considerable attention. A reduction in overall costs can only be realized if telemedicine serves as a substitute for higher cost services. Yet recent evidence suggests DTC telemedicine is unlikely to decrease healthcare costs, at least in the short term. ${ }^{20}$ A study of 300,000 enrollees in a California HMO found only $12 \%$ of telemedicine visits replaced in-person care and $88 \%$ represented new utilization. In our study, nearly three quarters of patients said they would have sought care at an urgent care/ retail clinic or physician's office had they not used DTC telemedicine, and only $6 \%$ said they would have gone to the ED. Importantly, however, $15 \%$ said they would have not sought care at all had the telemedicine service not been available. DTC telemedicine may therefore have the potential to increase healthcare spending by inducing some demand. Excessive utilization for low-acuity conditions has been documented previously in the context of retail clinics. ${ }^{21}$ Research has not borne out any substitution effect of retail clinics for patients with low-acuity conditions otherwise showing up in the ED. ${ }^{22}$ It simply may be the case that more healthcare availability is associated with more healthcare utilization, particularly when there are low barriers to accessing services. That said, increasing utilization is not necessarily a bad thing. Over $30 \%$ of patients in our sample did not report insurance information, suggesting some were probably uninsured. Increasing appropriate healthcare use among those with limited access is a potential benefit of DTC telemedicine.
Patients who used a coupon for services rated their provider and the telemedicine system more highly than those who did not. Given such financial incentives at the point of care rarely (if ever) exist in traditional healthcare settings, this was a unique opportunity to evaluate whether satisfaction varied by real time out of pocket cost. Patients who used a coupon may have had different expectations or motivations for accessing care which influenced their satisfaction ratings. Coupon users were more likely to access the system more than once. It is possible these patients initially accessed the telemedicine system because it was cheap or free and, then, having had a positive experience, sought additional care. Trends in utilization patterns by coupon use is an area warranting further study.

Patient satisfaction has been shown to be associated with physicians' drug prescribing practices. ${ }^{23,}{ }^{24} \mathrm{We}$ also found patient satisfaction was higher among those that received prescriptions. Patients may believe prescriptions are easier to access via telemedicine compared to traditional healthcare settings, as some DTC telemedicine platforms explicitly advertise their services a convenient way to get refills or new prescriptions. Patients seeking prescriptions which they ultimately did not receive may be particularly dissatisfied with their telemedicine experience. Major questions remain about the influence of a consumer-directed healthcare model like telemedicine on prescribing incentives. Simply giving patients prescriptions they want (but do not need) may result in higher satisfaction ratings, but results in lower quality. Indeed, substantial variation in the quality of telemedicine care has been reported. $^{25,26}$

To date, no study has described a large group of physicians who practice DTC telemedicine. The overwhelming majority of physicians in our sample were trained at US medical schools and were generalists. Foreign medical graduates now represent more than a quarter of the US physician workforce. ${ }^{27}$ Patients in our study were more satisfied with US-trained physicians, compared to their non-US-trained counterparts, which has been documented previously. ${ }^{28}$ That said, our findings may not be generalizable to non-telemedicine settings. Communication challenges due to language or cultural barriers may be exacerbated by the telemedicine medium, thereby affecting satisfaction ratings more than would be expected in traditional face-to-face care.

This study had some limitations. We were unable to directly measure the impact of telemedicine on other healthcare utilization, as we were reliant on patient selfreport of what they would have done had they not used telemedicine. Additionally, we had a limited amount of physician data, which we gleaned from publicly available sources. Many questions therefore remain regarding the composition of the telemedicine physician workforce. Finally, the OCG is one of numerous DTC telemedicine providers. Thus, it is unknown to what extent our findings regarding the OCG system are generalizable to other similar telemedicine platforms. 


\section{CONCLUSIONS}

DTC telemedicine is used for a wide array of conditions and appears to serve primarily as a substitute for retail clinics or urgent care. We found patient satisfaction to be high overall; however, it varied by coupon use and prescription receipt. Telemedicine offers benefits of timely access and high patient satisfaction, but impact on primary care and ED use is likely to be small under present usage patterns.

\section{Contributors Not Meeting Criteria for Authorship. None}

Prior Presentations. This work was presented at the Society of General Internal Medicine Annual Meeting in April 2017, as well as at the AcademyHealth Annual Research Meeting in June 2017.

Corresponding Author: Kathryn A. Martinez, PhD, MPH; Center for Value-Based Care Research Cleveland Clinic, Cleveland, OH, USA (e-mail: martink12@ccf.org).

\section{Compliance with Ethical Standards:}

This study was approved by the Institutional Review Board of the Cleveland Clinic.

Conflict of Interest: The authors declare that they do not have a conflict of interest.

\section{REFERENCES}

1. Kvedar J, Coye MJ, Everett W. Connected health: a review of technologies and strategies to improve patient care with telemedicine and telehealth. Health Aff. 2014;33(2):194-199. https://doi.org/10. 1377/hlthaff.2013.0992.

2. Dorsey ER, Topol EJ. State of telehealth. Campion EW, ed. $N$ Engl J Med. 2016;375(2):154-161. https://doi.org/10.1056/NEJMra1601705.

3. Japsen B. Doctors' virtual consults with patients to double by 2020 . Forbes. 2015. https://www.forbes.com/sites/brucejapsen/2015/08/ 09/as-telehealth-booms-doctor-video-consults-to-double-by-2020/ \#a02baa54f9bf.

4. Physician appointment wait times and Medicaid and Medicare acceptance rates. Irving, TX; 2014. https://www.merritthawkins.com/ uploadedFiles/MerrittHawkings/Surveys/mha2014waitsurvPDF.pdf.

5. Schoen C, Osborn R, Squires D, Doty MM. Access, affordability, and insurance complexity are often worse in the United States compared to ten other countries. Health Aff (Millwood). 2013;32(12):2205-2215. https://doi.org/10.1377/hlthaff.2013.0879.

6. Ray KN, Chari A V, Engberg J, Bertolet M, Mehrotra A. Opportunity costs of ambulatory medical care in the United States. Am J Manag Care. 2015;21(8):567-574. http://www.ncbi.nlm.nih.gov/pubmed/26295356. Accessed July 14, 2017.

7. Tai-Seale M, McGuire TG, Zhang $\mathbf{W}$. Time allocation in primary care office visits. Health Serv Res. 2007;42(5):1871-1894. https://doi.org/10. $1111 /$ j. 1475-6773.2006.00689.x.

8. Mehrotra A, Lave JR. Visits To retail clinics grew fourfold from 2007 to 2009, although their share of overall outpatient visits remains low. Health Aff. 2012;31(9):2123-2129. https://doi.org/10.1377/hlthaff.2011.1128.
9. Mehrotra A. The convenience revolution for treatment of low-acuity conditions. JAMA. 2013;310(1):35-36. https://doi.org/10.1001/jama. 2013.6825.

10. Gordon AS, Adamson WC, DeVries AR. Virtual visits for acute, nonurgent care: a claims analysis of episode-level utilization. $J$ Med Internet Res. 2017;19(2):e35. https://doi.org/10.2196/jmir.6783.

11. "Health policy brief: telehealth parity laws." Health Affairs. 2016.

12. Uscher-Pines L, Mehrotra A. Analysis of Teladoc use seems to indicate expanded access to care for patients without prior connection to a provider. Heal Affiars. 2014;33(2):258-264. https://doi.org/10.1377/ hlthaff.2013.0989.

13. Courneya PT, Palattao KJ, Gallagher JM. HealthPartners' online clinic for simple conditions delivers savings of $\mathbf{\$ 8 8}$ per episode and high patient approval. Health Aff. 2013;32(2):385-392. https://doi.org/10.1377/ hlthaff.2012.1157.

14. Polinski JM, Barker T, Gagliano N, Sussman A, Brennan TA, Shrank WH. Patients' satisfaction with and preference for telehealth visits. $J$ Gen Intern Med. 2016;31(3):269-275. https://doi.org/10.1007/s11606-0153489-x.

15. Kahn JM. Virtual visits-confronting the challenges of telemedicine. $N$ Engl J Med. 2015;372(18):1684-1685. https://doi.org/10.1056/ NEJMp1500533.

16. Giordano LA, Elliott MN, Goldstein E, Lehrman WG, Spencer PA. Development, implementation, and public reporting of the HCAHPS survey. Med Care Res Rev. 2010;67(1):27-37. https://doi.org/10.1177/ 1077558709341065.

17. Song Y, Skinner J, Bynum J, Sutherland J, Wennberg JE, Fisher ES. Regional variations in diagnostic practices. $N$ Engl J Med. 2010;363(1):45-53. https://doi.org/10.1056/NEJMsa0910881.

18. Mehrotra A, Paone S, Martich GD, Albert SM, Shevchik GJ. Characteristics of patients who seek care via eVisits instead of office visits. Telemed J E Health. 2013;19(7):515-519. https://doi.org/10.1089/tmj. 2012.0221.

19. Mehrotra A, Wang MC, Lave JR, Adams JL, McGlynn EA. Retail clinics, primary care physicians, and emergency departments: A comparison of patients' visits. Health Aff. 2008;27(5):1272-1282. https://doi.org/10. 1377/hlthaff.27.5.1272.

20. Ashwood JS, Mehrotra A, Cowling D, Uscher-Pines L. Direct-toconsumer telehealth may increase access to care but does not decrease spending. Health Aff. 2017;36(3):485-491. https://doi.org/10.1377/ hlthaff.2016.1130.

21. Ashwood JS, Gaynor M, Setodji CM, Reid RO, Weber E, Mehrotra A. Retail clinic visits for low-acuity conditions increase utilization and spending. Health Aff. 2016;35(3):449-455. https://doi.org/10.1377/ hlthaff.2015.0995.

22. Martsolf G, Fingar KR, Coffey R, et al. Association between the opening of retail clinics and low-acuity emergency department visits. Ann Emerg Med. 2017;69(4):397-403.e5. https://doi.org/10.1016/j.annemergmed. 2016.08.462.

23. Fenton JJ, Jerant AF, Bertakis KD, Franks P. The cost of satisfaction: a national study of patient satisfaction, health care utilization, expenditures, and mortality. Arch Intern Med. 2012;172(5):405-411. https://doi. org/10.1001/archinternmed.2011.1662.

24. Miller M, Miller M, Rabago D. Patient satisfaction, prescription drug abuse, and potential unintended consequences. JAMA. 2012;307(13):1377. https://doi.org/10.1001/jama.2012.419.

25. Schoenfeld AJ, Davies JM, Marafino BJ, et al. Variation in quality of urgent health care provided during commercial virtual visits. JAMA Intern Med. 2016;176(5):635. https://doi.org/10.1001/jamainternmed.2015. 8248.

26. Uscher-Pines L, Mulcahy A, Cowling D, Hunter G, Burns R, Mehrotra A. Access and quality of care in direct-to-consumer telemedicine. Telemed e-Health. 2016;22(4):282-287. https://doi.org/10.1089/tmj.2015.0079.

27. Brotherton SE, Etzel SI. Graduate medical education, 2014-2015. JAMA. 2015;314(22):2436. https://doi.org/10.1001/jama.2015.10473.

28. Howard DL, Bunch CD, Mundia wo, et al. Comparing United States versus international medical school graduate physicians who serve African-American and White elderly. Health Serv Res. 2006;41(6):21552181. https://doi.org/10.1111/j.1475-6773.2006.00587.x. 